\title{
SURVEILLANCE INTER-ÉPIDÉMIQUE DU FOYER LEISHMANIEN de Keur Moussa (Thiès, Sénégal)
}

\author{
NIANG A.A.*, TROUILLET J.** \& FAYE O.**
}

Summary : INTER-EPIDEMIC SUPERVISION OF THE LEISHMANIASIS FOCUS of Keur Moussa (Thies, Senegal)

A supervision of the focus of human cutaneous leishmaniasis of Keur Moussa has been carried out in 1988-1989 and in 19911992. Among the 13 species gathering the 10,144 phlebotomine sandflies traped, two belong to genus Phlebotomus, the others to genus Sergentomyia. Phlebotomus duboscai, that has been found out by Deded et al. in 1980 as the vector of this leishmaniasis in Senegal, is the most represented species after Sergentomyia schwetzi, with respectively 32.3 and $28.5 \%$ of the fauna during these two periods. It is twice more abundant and frequent in the monastery area than the religious one. This difference may be due to the better micro-climatic conditions, the abundance of rodents and the human proximity. After the rainy saison that influence a lot $P$. duboscai's activity, the density of phlebotomine sandflies makes a progressive raise, as well as the temperature and humidity, to reach two maxima in April-May and July-August. Parasitological studies on females of phlebotomine sandflies and rodents Arvicanthis niloticus, Mastomys erythroleucus and Cricetomys gambianus are negative. This focus seems to be again in an inter-epidemic phase.

KEY WORDS : Leishmaniasis, epidemiology, Phlebotomus duboscqi, Senegal.

\section{Résumé :}

Une surveillance du foyer de leishmaniose cutanée humaine de Keur Moussa a été menée en 1988-1989 et en 1991-1992. Les 10144 phlébotomes récoltés sont répartis entre 13 espèces dont deux sont du genre Phlebotomus, les autres du genre Sergentomyia. Phlebotomus duboscai, dont le rôle vecteur de cette leishmaniose a été mis en évidence au Sénégal par Dedet et al. en 1980, est l'espèce la plus représentée, derrière Sergentomyia schwetzi, avec respectivement 32,3 et 28,5\% du peuplement durant ces périodes. II est deux fois plus abondant et plus fréquent au domaine du monastère qu'au domaine des religieuses du fait des conditions micro-climatiques et phytogéographiques plus favorables, de l'abondance des rongeurs et de la présence plus constante de l'homme. Après l'arrêt des précipitations qui influent beaucoup sur l'activité de $\mathrm{P}$. duboscai, la densité des phlébotomes augmente progressivement en même temps que la température et l'hygrométrie pour atteindre deux maxima (en avril-mai et juillet-août). L'étude parasitologique s'est révélée négative chez les femelles de P. duboscqi et chez les rongeurs Arvicanthis niloticus, Mastomys erythroleucus et Cricetomys gambianus. Le foyer semble être entré à nouveau dans une phase inter-épidémique.

MOTS CLÉS : Leishmaniose, épidémiologie, Phlebotomus duboscqi, Sénégal.

\section{INTRODUCTION}

L e foyer de leishmaniose cutanée de Keur Moussa (Région de Thiès, Sénégal) a fait l'objet d'une étude suivie de 1976 à 1983 (Dedet et al., 1978; $1979 a, b, c, d ; 1980 ; 1981 ; 1982$; Desjeux et al., 1981; Desjeux et Dedet, 1982). Durant cette période d'observation, le rôle vecteur de Phlebotomus duboscqi NeveuLemaire, 1906 est mis en évidence pour la première fois au Sénégal, de même que celui de l'agent éthiologique : Leishmania major; le nombre de cas humains

\footnotetext{
* Laboratoire de Zoologie des Invertébrés Terrestres, Institut Fondamental d'Afrique Noire Cheikh Anta Diop, Université Cheikh Anta Diop, BP 206, Dakar, Sénégal.

** Laboratoire d'Écologie Parasitaire, Département de Biologie Animale, Faculté des Sciences et Techniques, Université Cheikh Anta Diop, BP 5005, Dakar, Sénégal.

Correspondance : Abdoul Aziz Niang.
}

n'a cessé de décroître, de 85 en 1976, 34 en 1977, neuf en 1978, deux en 1979, pour devenir nul en 1980 et 1981. La dernière surveillance a été levée en avril 1983 (Blanchot et al., 1984). Depuis, la maladie réapparaît périodiquement quand les populations de rongeurs-réservoirs augmentent. Par ailleurs, l'affection demeure sporadiquement présente au Sénégal. Le caractère endémo-épidémique de cette affection rend nécessaire l'établissement de surveillances régulières afin de prévenir de nouvelles flambées épidémiques. Depuis 1988, pendant deux périodes, nous avons étudié les fluctuations saisonnières de la faune phlébotomienne de ce foyer en général et du vecteur Pblebotomus duboscqi en particulier. Les rongeurs réservoirs, Arvicanthis niloticus, Mastomys erythroleucus et Tatera gambiana (Larivière et al., $1965 c$ et $d$, Ranque et Camerlynk, 1966; Ranque et al., 1974; Dedet et al., $1979 b ; 1981$ ), ont également été suivis et l'agent Leishmania major recherché chez ces hôtes et chez l'homme. 


\section{MATÉRIEL ET MÉTHODE}

\section{STATIONS DE CAPTURE}

a région de Keur Moussa, si bien décrite par Dedet et al. (1979a), est à environ $50 \mathrm{~km}$ sur l'axe Dakar-Thiès. La zone d'étude comprend deux stations séparées d'environ $500 \mathrm{~m}$ : le domaine du Monastère (station 1) renfermant des jardins, des vergers, ainsi que des terrains de friche, des champs et la résidence des religieuses (station 2) un petit verger. Les captures ont été réalisées à proximité des habitations, à l'entrée des terriers de rongeurs (Crycetomys gambianus et Mastomys erythroleucus) et dans les mottes de pailles, abris des Arvicanthis.

Le climat de la région cap-verdienne est caractérisé par l'alternance des alizés et de la mousson qui le découpe en deux saisons bien tranchées:

- une saison sèche (novembre à mai) marquée par la circulation de l'alizé continental desséchant adouci par l'alizé maritime de direction nord-ouest à nord-est avec souvent en janvier-février quelques pluies de " heug ", liées aux invasions d'air polaire;

- une saison humide ou hivernage (juin à octobre) durant laquelle on note le remplacement des alizés par la mousson, flux chaud et humide entraînant des pluies irrégulières et orageuses.

Les températures moyennes mensuelles oscillent entre 21-22 ${ }^{\circ} \mathrm{C}$ (janvier-février) et $27-28{ }^{\circ} \mathrm{C}$ (septembreoctobre). Comme pour l'ensemble de la presqu'île, elles sont influencées, de même que l'humidité relative constamment élevée (40 à $80 \%$ ), par la proximité de la mer. Le vent, généralement de direction nordouest à nord-est, peut atteindre des vitesses de 4 à $6 \mathrm{~m} / \mathrm{s}$. La hauteur des précipitations varie beaucoup d'une année à l'autre (150 à $1000 \mathrm{~mm}$ ), pour atteindre une moyenne annuelle un peu inférieure à $500 \mathrm{~mm}$. L'hivernage de 1988 a été assez bien arrosé (555,5 mm) tandis qu'en 1990 et 1991, les précipitations ont été faibles (408,2 et $333,5 \mathrm{~mm}$ ).

\section{MÉTHODES D'ÉTUDE}

Les échantillonnages des phlébotomes ont été réalisés de janvier 1988 à janvier 1989 au rythme d'une séance hebdomadaire et de mars 1991 à février 1992 à raison de cinq jours par mois (du lundi au vendredi).

Six techniques de piégeage sont utilisées au cours de séances nocturnes de 19 heures à 8 heures. Les trois premières sont à visée phénologiques :

1) le piégeage adhésif à l'aide des papiers imbibés d'huile de ricin et insérés à l'entrée des terriers ou accrochés sur deux baguettes en bois en forme de $T$ (pour le piégeage péri-domiciliaire);
2) les pièges lumineux " $\mathrm{CDC}$ miniature light trap " suspendus à l'entrée des terriers et à l'intérieur des habitations;

3) la capture manuelle sur paroi verticale avec un aspirateur-nasse et une lampe de poche, qui fournit la plupart des femelles gravides ou gorgées destinées à l'élevage;

4) une guirlande lumineuse suspendue au monastère, près du noviciat, face aux jardins.

Les autres techniques de piégeage sont à visée éthologique :

5) la capture manuelle sur appât humain pour l'étude de l'anthropophilie;

6) le piège adhésif lumineux pour l'étude du phototactisme (cube en bois de $20 \mathrm{~cm}$ de côté avec trois faces en bois et les trois autres recouvertes de papiers huilés avec une source lumineuse interne de faible intensité).

Lors du dépouillement, tous les échantillons mâles ainsi que les femelles mortes sont conservés dans de l'alcool à $70^{\circ}$ jusqu'à leur montage et leur identification spécifique. Les femelles capturées vivantes sont mises en élevage, engorgées sur hôte rongeur ou disséquées en milieu stérile.

La recherche d'infestation spontanée par des formes promastigotes se fait au niveau du tube digestif. En cas de positivité, le tube digestif est inoculé à un milieu de culture NNN qui est ensuite mis en incubation à $24{ }^{\circ} \mathrm{C}$. La négativité est prononcée après trois repiquages (un par semaine); s'il s'agit bien de leishmanies, les repiquages sont poursuivis tous les 15 jours. Des séances de capture de rongeurs ont été effectuées tous les deux mois à l'entrée des différents terriers afin d'identifier les principaux hôtes des phlébotomes et de dépister la présence de lésions chez ces réservoirs potentiels de leishmanies. Ces animaux sont utilisés pour faire engorger les phlébotomes femelles capturés à jeun.

\section{RÉSULTATS}

\section{RicheSSE ET ABONDANCE DES PHLÉBOTOMES}

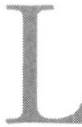
es prospections réalisées au niveau du foyer leishmanien de Keur Moussa entre janvier 1988 et janvier 1989 puis entre mars 1991 à février 1992 ont permis la capture de 10144 phlébotomes répartis entre 13 espèces qui sont :

Phlebotomus (Phlebotomus) duboscqi Neveu-Lemaire, 1906;

P. (Anaphlebotomus) rodhaini Parrot, 1930;

Sergentomyia (Grassomyia) inermis (Theodor, 1938);

S. (Grassomyia) squamipleuris (Newstead, 1912);

S. (Sintonius) adleri (Theodor, 1933);

S. (Sintonius) clydei (Sinton, 1928); 
S. (Sergentomyia) antennata (Newstead, 1912);

S. (Sergentomyia) buxtoni (Theodor, 1933);

$S$. (Sergentomyia) dubia (Parrot, Mornet et Cadenat, 1945);

S. (Sergentomyia) schwetzi (Adler, Theodor et Parrot, 1929);

S. (Parrotomyia) africana africana (Newstead, 1912);

S. (Parrotomyia) freetownensis (Sinton, 1930);

S. (Parrotomyia) magna (Sinton, 1932).

- Abondance tous piégeages confondus

L'analyse de l'ensemble des récoltes (tableau I) montre que deux espèces sont nettement plus abondantes que les autres : $S$. schwetzi représentant près de la moitié des effectifs ( $42 \%$ en 1988 et $47,90 \%$ en 1991) et $P$. duboscqi, le vecteur de la leishmaniose cutanée humaine au Sénégal (avec 32,30 et 28,50 \% en 1989 et 1991). Par contre, quatre espèces ne sont représentées que par quelques individus : S. buxtoni, $S$. inermis, $S$. freetownensis et $P$. rodhaini. Ces deux dernières, de même que $S$. africana africana, n'ont été capturées qu'en 1991, alors que les deux spécimens de S. inermis ont été récoltés en 1988.

\begin{tabular}{|c|c|c|c|c|}
\hline \multirow[b]{2}{*}{ Espèces } & \multicolumn{2}{|c|}{$\begin{array}{c}\text { Janvier } 1988 \\
\text { à mars } 1989\end{array}$} & \multicolumn{2}{|c|}{$\begin{array}{c}\text { Mars } 1991 \\
\text { à février } 1992\end{array}$} \\
\hline & Nombre & $\begin{array}{c}\text { Abondance } \\
(\%)\end{array}$ & Nombre & $\begin{array}{c}\text { Abondance } \\
(\%)\end{array}$ \\
\hline S. schwetzi & 1742 & 42,00 & 2876 & 47,9 \\
\hline P. duboscqi & 1337 & 32,30 & 1711 & 28,5 \\
\hline S. magna & 269 & 6,49 & 451 & 7,5 \\
\hline S. dubia & 232 & 5,62 & 443 & 7,4 \\
\hline S. clydei & 376 & 9,07 & 99 & 1,6 \\
\hline S. adleri & 69 & 1,67 & 190 & 3,2 \\
\hline S. antennata & 72 & 1,74 & 101 & 1,7 \\
\hline S. squamipleuris & 35 & 0,84 & 1 & 0,02 \\
\hline S. buxtoni & 9 & 0,22 & 18 & 0,3 \\
\hline S. africana africana & - & - & 94 & 1,6 \\
\hline S. freetownensis & - & - & 12 & 0,2 \\
\hline P. rodbaini & - & - & 5 & 0,08 \\
\hline S. inermis & 2 & 0,05 & - & - \\
\hline Total & 4143 & 100 & 6001 & 100 \\
\hline
\end{tabular}

Test du $\chi^{2}$ pour les nombres de phlébotomes aux deux périodes : $\mathrm{ddl}=7, \chi^{2}=1566,8$ significatif à $5 \%$ d'erreur.

Tableau I. - Ensemble des récoltes à Keur Moussa tous piègeages confondus.

S. magna et $S$. dubia sont légèrement mieux représentés pendant la deuxième période alors que $S$. clydei s'est considérablement réduit passant de 9,07\% en 1988 à 1,60\% en 1991. La même remarque peut être faite pour $S$. squamipleuris dont un seul échantillon est rencontré en 1991 contre 35 en 1988.

- Abondance des phlébotomes selon les stations

La comparaison des captures obtenues au niveau du Monastère et au niveau du domaine des religieuses (tableau II) met en évidence l'abondance de $P$. duboscqi deux fois plus importante à la première station. Par contre, les six autres espèces les mieux représentées, S. schwetzi S. magna, S. dubia, S. clydei, S. adleri et $S$. antennata, sont plus abondantes à la station 2.

Une explication peut se trouver dans le fait que les jardins et vergers des moines sont beaucoup plus développés que ceux des religieuses, il s'en suit une présence plus grande des hommes et des rongeurs, conditions qui favorisent $P$. duboscqi, alors que la station 2 voit les espèces moins péri-domestiques plus présentes.

\begin{tabular}{|c|c|c|c|c|c|c|}
\hline \multirow[b]{2}{*}{ Espèces } & \multicolumn{2}{|c|}{ Station 1} & \multicolumn{2}{|c|}{ Station 2} & \multicolumn{2}{|c|}{ Total } \\
\hline & nombre & $\%$ & nombre & $\%$ & nombre & e \% \\
\hline S. schwetzi & 2253 & 41,23 & 2365 & 50,54 & 4618 & 45,52 \\
\hline P. duboscqi & 2181 & 39,91 & 867 & 18,53 & 3048 & 30,05 \\
\hline S. magna & 328 & 6,00 & 392 & 8,38 & 720 & 7,10 \\
\hline S. dubia & 243 & 4,45 & 432 & 9,23 & 675 & 6,65 \\
\hline S. clydei & 156 & 2,85 & 319 & 6,82 & 475 & 4,68 \\
\hline S. adleri & 134 & 2,45 & 125 & 2,67 & 259 & 2,55 \\
\hline S. antennata & 73 & 1,34 & 100 & 2,14 & 173 & 1,71 \\
\hline S. africana & 54 & 0,99 & 40 & 0,85 & 94 & 0,93 \\
\hline P. squamipleuris & 21 & 0,38 & 15 & 0,32 & 36 & 0,35 \\
\hline S. buxtoni & 18 & 0,33 & 9 & 0,19 & 27 & 0,27 \\
\hline S. freetownensis & 3 & 0,05 & 9 & 0,19 & 12 & 0,12 \\
\hline$P$ rodhaini & 0 & 0,00 & 5 & 0,11 & 5 & 0,05 \\
\hline S. inermis & 1 & 0,02 & 1 & 0,02 & 2 & 0,02 \\
\hline Total & 5465 & 100 & 4679 & 100 & 10144 & 100 \\
\hline
\end{tabular}

Nombres de phlébotomes aux stations 1 et $2: \mathrm{ddl}=9, \chi^{2}=2133$ significatif à $5 \%$ d'erreur.

Tableau II. - Abondance selon les stations des phlébotomes récoltés à Keur Moussa.

- Abondance des phlébotomes selon les types de pièges

L'analyse de l'abondance des récoltes en fonction du mode de piégeage (tableau III) n'appelle qu'une remarque pour $P$. duboscqi : les pièges CDC placés à proximité des habitations recueillent moins de spécimens que les autres types de captures. Ceci est sans doute lié aux déplacements très saisonniers de cette espèce, qui ne doit s'éloigner des terriers de rongeurs (gîte larvaire et gite de repos) que lorsque les conditions lui sont optimales. Toutefois, la guirlande éclairée placée près du noviciat a recueilli une bonne proportion de $P$. duboscqi, du fait sans doute de la proximité et de l'abondance des terriers dans ce secteur.

$S$. squamipleuris est beaucoup plus fréquent sur la guirlande éclairée où elle représente $7 \%$ des captures au lieu de $1 \%$ dans les autres types de pièges. Cette espèce a un biotope assez particulier vivant le plus souvent dans les broussailles, les buissons, sous le couvert des bois, dans les amas de feuilles mortes. Les Grassomyia sont fortement attirés par la lumière. 


\begin{tabular}{|c|c|c|c|c|c|c|c|c|}
\hline \multirow[b]{2}{*}{ Espèces } & \multicolumn{2}{|c|}{ Papiers huilés } & \multicolumn{2}{|c|}{$\begin{array}{c}\text { Pièges CDC } \\
\text { (terriers) }\end{array}$} & \multicolumn{2}{|c|}{$\begin{array}{l}\text { Pièges CDC } \\
\text { (habitations) }\end{array}$} & \multicolumn{2}{|c|}{ Guirlande éclairée } \\
\hline & nombre & $\%$ & nombre & $\%$ & nombre & $\%$ & nombre & $\%$ \\
\hline S. schwetzi & 4025 & 45,13 & 338 & 56,62 & 121 & 47,08 & 74 & 32,17 \\
\hline P. duboscqi & 2879 & 32,28 & 101 & 16,92 & 22 & 8,56 & 46 & 20,00 \\
\hline S. magna & 634 & 7,11 & 35 & 5,86 & 20 & 7,78 & 29 & 12,61 \\
\hline S. dubia & 470 & 5,27 & 60 & 10,05 & 32 & 12,45 & 40 & 17,39 \\
\hline S. clydei & 362 & 4,06 & 47 & 7,87 & 50 & 19,46 & 16 & 6,96 \\
\hline S. adleri & 238 & 2,67 & 6 & 1,01 & 6 & 2,33 & 4 & 1,74 \\
\hline S. antennata & 163 & 1,83 & 3 & 0,50 & 3 & 1,17 & 3 & 1,30 \\
\hline S. africana africana & 93 & 1,04 & 1 & 0,17 & - & & - & \\
\hline P. squamipleuris & 14 & 0,16 & 4 & 0,67 & 2 & 0,78 & 16 & 6,96 \\
\hline S. buxtoni & 23 & 0,26 & 2 & 0,34 & 1 & 0,39 & 1 & 0,43 \\
\hline S. freetownensis & 12 & 0,13 & - & & - & & - & \\
\hline P. rodhaini & 5 & 0,06 & - & & - & & - & \\
\hline S. inermis & 1 & 0,01 & - & & - & & 1 & 0,43 \\
\hline Total & 8919 & 100 & 597 & 100 & 257 & 100 & 230 & 100 \\
\hline
\end{tabular}

Test du $\chi^{2}$ pour les nombres des trois premières espèces : $\mathrm{ddl}=4, \chi^{2}=197,3$ significatif à $5 \%$ d'erreur.

Tableau III. - Abondance des phlébotomes selon les quatre principaux modes de piègeage.

S. clydei est plus recueilli à proximité des habitations du fait de son comportement trophique polyvalent et son anthropophilie.

Les deux espèces $S$. freetownensis et $P$. rodhaini ne sont rencontrées quà l'entrée des terriers.

- Abondance de P. duboscqi selon les terriers

$P$. duboscqi est la seule espèce à être impliquée dans l'épidémiologie de la leishmaniose cutanée humaine. Aussi apparut-il intéressant d'étudier son abondance en fonction des types de terriers classés suivant leurs occupants (espèces de rongeurs et reptiles). Cette analyse est faite sur la base du piègeage adhésif effectué pendant la période 1991-1992. Les résultats figurent dans le tableau IV.

Les terriers de Cricetomys sans ou avec reptiles ont fourni la grande majorité $(93,3 \%)$ des $P$. duboscqi capturés. La moyenne d'insectes par terrier est plus élevée au niveau de la station 1 du fait probablement de l'abondance des rongeurs liée au microclimat plus favorable du Monastère sous l'influence des jardins et vergers. Les terriers de Cricetomys fréquentés par des reptiles sont les plus productifs.

Seuls $14 P$. duboscqi (soit $0,8 \%$ du total) ont été capturés dans le secteur des Arvicanthis. Les nids en surface sont sans aucune protection contre les pluies hivernales; les animaux sont obligés de les abandonner pendant cette saison. Aucun insecte n'y a été capturé entre août et janvier. Les terriers colonisés par des reptiles n'ont fourni que $0,6 \%$ des $P$. duboscqi piégés et ceux non identifiés, $0,3 \%$.

Le test du $\chi^{2}$, significatif aussi bien avec les totaux qu'avec les moyennes par terrier, confirme qu'au niveau de Keur Moussa, $P$. duboscqi est particulièrement inféodé aux terriers de Cricetomys.

\section{FRÉQUENCE}

La fréquence des espèces est calculée à partir des récoltes réalisées avec les deux principales techniques : les papiers huilés et les pièges " $\mathrm{CDC}$ ". Les résultats figurent dans les tableaux V et VI.

\begin{tabular}{|c|c|c|c|c|c|}
\hline \multirow[b]{3}{*}{ Terriers de } & \multicolumn{5}{|c|}{ Nombre de phlébotomes } \\
\hline & \multicolumn{2}{|c|}{ Station 1} & \multicolumn{2}{|c|}{ Station 2} & \multirow[b]{2}{*}{ Tota } \\
\hline & nombre & nb./terrier & nombre & nb./terrier & \\
\hline Cricetomys * & 1010 & 16,6 & 237 & 5,5 & 1247 \\
\hline Cricetomys (+Reptiles) * & 373 & 21,9 & 18 & 9 & 391 \\
\hline Arvicanthis + Mastomys & 14 & 2,8 & - & - & 14 \\
\hline Reptile * & 2 & 2 & 8 & 4 & 10 \\
\hline Non identifiés & 1 & 1 & 4 & 0,7 & 5 \\
\hline Total & 1400 & 16,5 & 267 & 5 & 1667 \\
\hline
\end{tabular}

Nombres de phlébotomes des lignes ${ }^{*}: \mathrm{ddl}=1, \chi^{2}=9522,6$ significatif à $5 \%$ d'erreur.

Tableau IV. - Abondance selon les terriers de P. duboscqi (à l'aide du piégeage adhésif). 


\begin{tabular}{|c|c|c|c|c|c|c|}
\hline & \multicolumn{3}{|c|}{ Piégeage adhésif } & \multicolumn{3}{|c|}{ Piégeage lumineux } \\
\hline & Station 1 & Station 2 & Total & Station 1 & Station 2 & Total \\
\hline S. schwetzi & 90,4 & 98,1 & 98,1 & 41,5 & 48,1 & 61,5 \\
\hline P. duboscqi & 78,8 & 78,8 & 86,5 & 21,9 & 21,1 & 32,7 \\
\hline S. magna & 21,1 & 55,8 & 73,1 & 12,2 & 15,4 & 21,1 \\
\hline S. dubia & 30,8 & 44,2 & 51,9 & 14,6 & 23,1 & 32,7 \\
\hline S. clydei & 42,3 & 59,6 & 63,5 & 19,5 & 23,1 & 32,7 \\
\hline S. adleri & 7,7 & 46,1 & 50,0 & 7,3 & 3,8 & 9,6 \\
\hline S. antennata & 23,1 & 28,8 & 40,4 & 0 & 3,8 & 3,8 \\
\hline S. buxtoni & 5,8 & 3,8 & 9,6 & - & 3,8 & 3,8 \\
\hline S. squamipleuris & 1,9 & 5,8 & 7,7 & 4,9 & 1,9 & 5,8 \\
\hline S. inermis & - & 1,9 & 1,9 & - & - & - \\
\hline
\end{tabular}

Tableau V. - Fréquences des espèces à Keur Moussa en 1988-1989.

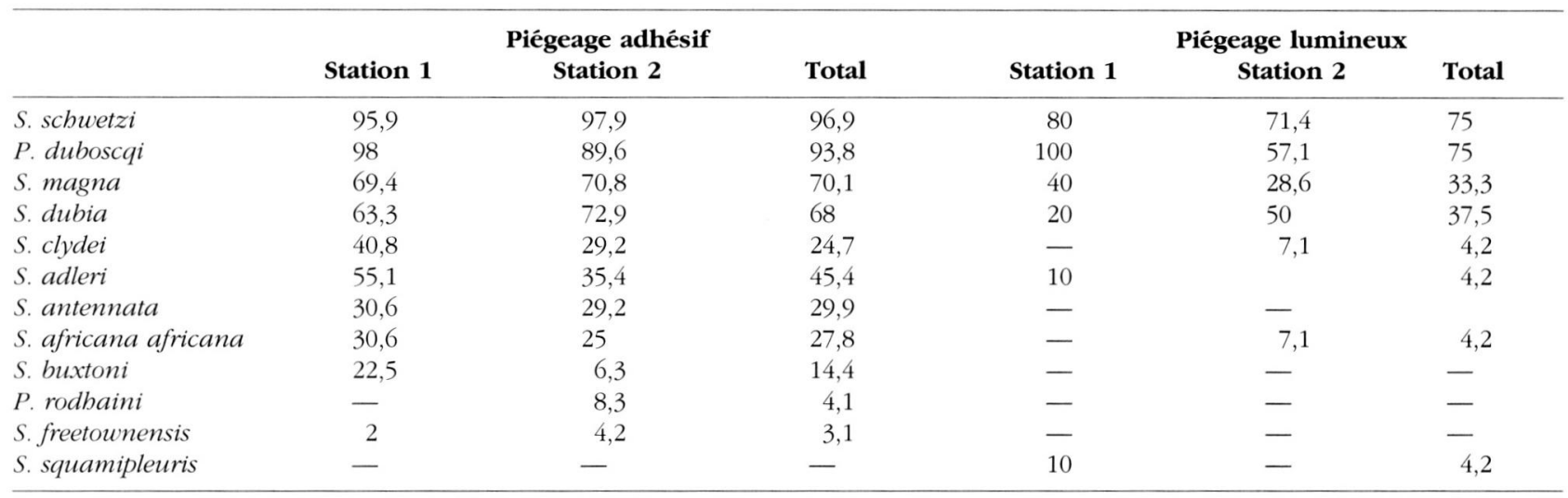

Tableau VI. - Fréquences des espèces à Keur Moussa en 1991-1992.

Pour la période 1988-1989 et particulièrement avec les papiers huilés, on retrouve un classement très proche de celui obtenu pour l'abondance, les deux espèces les plus abondantes dans les deux stations se révélant les plus fréquentes: $S$. schwetzi et $P$. duboscqi. On peut toutefois remarquer que $S$. magna, un peu moins abondant, est aussi fréquent que $S$. clydei. S. adleri est plus fréquent que $S$. antennata, alors que leurs abondances sont voisines. Les écarts de fréquence entre les deux stations sont assez faibles pour la plupart des espèces. Ils sont conséquents pour $S$. adleri, $S$. clydei et $S$. magna qui sont nettement plus fréquents à la station 2 .

Avec le piégeage lumineux, aucune espèce n'est très commune dans l'une ou l'autre des stations. Seule $S$. schwetzi a une fréquence de plus de $50 \%$ sur l'ensemble des récoltes.

En 1991-1992, avec le piégeage adhésif, seules les quatre espèces les plus abondantes sont très communes $^{1}$ (S. schwetzi, $P$. duboscqi, S. magna et

1. La fréquence d'une espèce est le pourcentage des captures positives sur le total des captures. Une espèce peut être :

- très commune : fréquence supérieure à $50 \%$;

- commune : fréquence comprise entre 25 et $50 \%$;

- rare : fréquence comprise entre 10 et $25 \%$;

- très rare : fréquence inférieure à $10 \%$.
S. dubia), quatre sont communes (S. clydei, S. adleri, $S$. antennata et $S$. africana africana), $S$. buxtoni est rare, $P$. rodhaini et $S$. freetownensis sont très rares. Pendant cette même période, $P$. duboscqi est rencontré à toutes les séances de piègeage lumineux au niveau du Monastère alors qu'au niveau du domaine des religieuses, cette fréquence est moins importante (57\%). Cette différence peut être attribuée aux conditions d'humidité et de température favorables, liées à la phytogéographie plus fournie de la station 1 , à l'abondance permanente des rongeurs et la présence plus constante de l'homme, autant de paramètres qui favorisent le déplacement de cette espèce. $S$. schwetzi est la seule autre espèce très fréquente dans ce type de piège. $S$. magna et $S$. dubia, très communs précédemment avec le piégeage adhésif, y sont communs. Les autres espèces ont très rarement ou pas été rencontrées avec le piège lumineux.

\section{VARIATIONS SAISONNIÈRES D'ABONDANCES}

Cette étude est basée sur les résultats du piègeage adhésif, les autres techniques de capture apportant des renseignements complémentaires.

L'activité des phébotomes s'est poursuivie tout au long des deux périodes de l'étude avec des fluctuations importantes en fonction des saisons. 


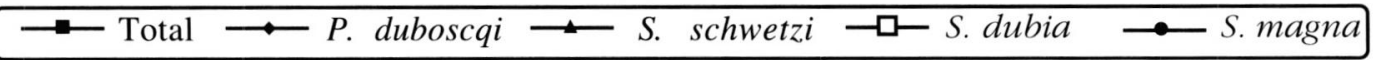
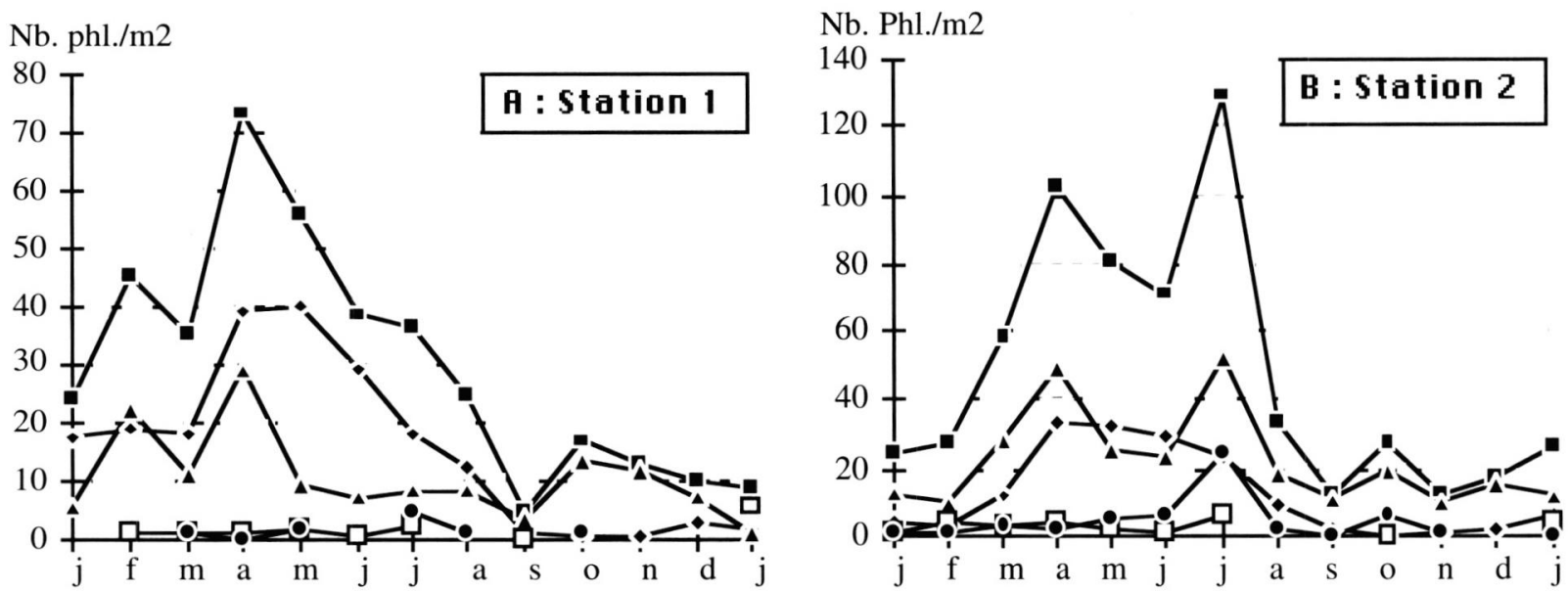

Fig. 1A et B. - Variations saisonnières d'abondances du peuplement de phlébotomes et des principales espèces en 1988-1989.

Dans la période 1998-1989, l'ensemble du peuplement de la station 2 présente une courbe de fluctuations à trois pics, en février, avril et octobre (fig. 1A). Le premier se situe au moment des minima de la température et de l'hygrométrie, le second durant la phase ascendante de ces deux paramètres climatiques et le troisième, beaucoup moins marqué, en fin de saison des pluies; pendant cette dernière période, l'abondance des phlébotomes serait sans doute liée à la reprise de leur activité consécutive à l'arrêt des précipitations. Pour la station 2, les pics se situent en avril, juillet (début de saison des pluies) et octobre (fig. 1B).

$P$. duboscqi est présent toute l'année dans les terriers. On relève un acroissement important de ses populations dès le mois de mars, parallèle à l'augmentation de la température et de l'hygrométrie. Elles atteignent leur maximum en avril-mai et amorcent une régression dès le mois de juin, pour connaître leur minimum en septembre-octobre-novembre. $S$. schwetzi présente les mêmes variations que $P$. duboscqi à part la régression amorcée plus tôt (en mai) à la station 1. Pour les autres espèces, les proportions sont si faibles qu'il est difficile d'interpréter leurs variations.

Dans la deuxième phase de l'étude (1991-1992), la faune phlébotomienne est également active durant toute l'année. Par contre, la courbe d'abondance de l'ensemble des espèces (fig. 2A et B) est deux fois moins importante (ce qui peut être attribué aux différences de précipitations) et les trois pics sont un peu décalés par rapport à la première période : mai, août et novembre pour la station 1 et mai, août et octobre pour la station 2 .

Pour $P$. duboscqi constamment présent à l'entrée des terriers, les plus fortes densités sont notées entre mai et août après une augmentation progressive, mais irrégulière, entre mars et juillet à la station 1 , mars et mai à la station 2 où elles sont beaucoup moins importantes et où les effectifs de $S$. schwetzi dominent nettement sur les autres espèces avec des variations identiques à celles de l'ensemble du peuplement. P. duboscqi semble extrêmement sensible aux précipitations : avec les premières pluies ses populations diminuent immédiatement de façon spectaculaire aboutissant à de faibles densités en octobre-novembre. Le test de corrélation des moyennes mensuelles avec la pluviométrie, avec un décalage d'un mois est négativement significatif (coefficient $\mathrm{R}=-0,758$ à $5 \%$ d'erreur); ce qui laisse supposer que les fortes précipitations, du fait du lessivage des gîtes larvaires, entraîneraient, après un certain délai, une diminution des effectifs.

\section{FAUNE DE RONGEURS}

La faune de rongeurs de Keur Moussa est composée de 12 espèces parmi lesquelles on trouve : Cricetomys gambianus (rat de Gambie), Xerus erythropus (écureuil terrestre), Tatera gambiana, Arvicanthis niloticus et Mastomys erythroleucus (Duplantier et Granjon, 1990). A l'entrée de terriers, siège de notre étude, nous avons capturé des Cricetomys, des Arvicanthis et des Mastomys. Cricetomys gambianus occupe le plus grand nombre de terriers (104 parmi les 138 recensés) et compte plus d'individus, donc d'hôtes disponibles; mais cette espèce est apparue réfractaire aux souches de leishmanies isolées (Larivière, 1966). Les espèces réservoirs de base de Leishmania major, Arvicanthis niloticus (Larivière et al., $1965 c$ et $d$; Ranque et al., 1972 et 1974), Mastomys erythroleucus et Tatera gam- 


\section{$\longrightarrow$ Total $\multimap$ P. duboscqi $\longrightarrow$ S. schwetzi $\multimap-$ S. dubia $\multimap$-S. magna}
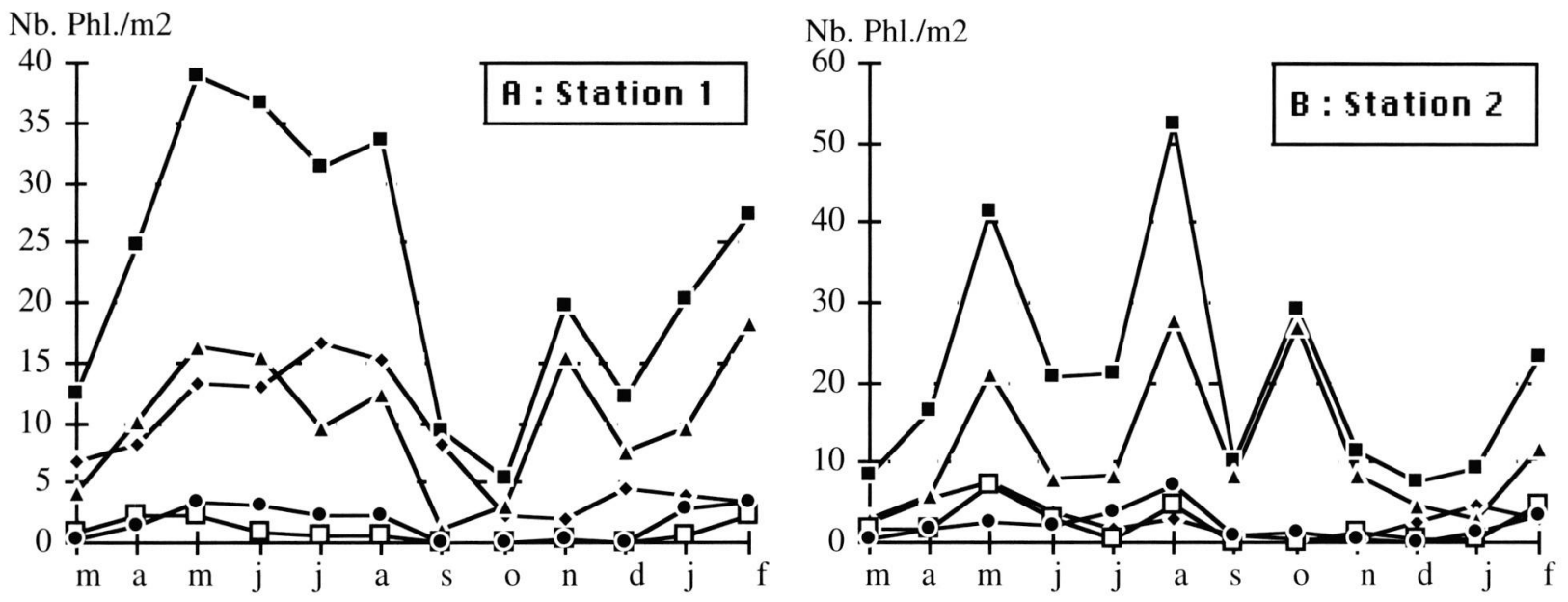

Fig. 2 A et B. - Variations saisonnières d'abondances du peuplement de phlébotomes et des principales espèces en 1991-1992.

biana (Dedet et al., 1981), sont faiblement représentées ou actuellement absentes (Tatera sur notre site) d'étude.

\section{ÉTUDE PARASITOLOGIQUE}

Entre mars 1991 et février 1992, nous avons disséqué 1907 phlébotomes dont 493 P. duboscqi parmi les 511 capturés. Seules deux femelles de $P$. duboscqi ont été trouvées porteuses de promastigotes qui toutefois n'ont pu être identifiés.

Nous avons également examiné 18 rongeurs dont 11 Arvicanthis niloticus et sept Mastomys erythroleucus. Les animaux ne portaient pas de lésions cutanées et les cultures de portions de leur rate ont été négatives. Pour ce qui est de l'affection humaine, l'examen des cas recensés par les Sours infirmières et la mise en culture de prélèvements à partir des lésions n'ont pas permis de déceler une quelconque infestation.

\section{DISCUSSION}

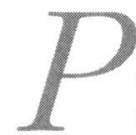

duboscqi est réparti en Afrique d'Est en Ouest sur une large bande de $20^{\circ}$ au nord de l'équateur. Les plus fortes densités sont cependant rencontrées dans les régions occidentales : Niger, Nigeria, Mali, Mauritanie, Sénégal, Gambie, Togo, Burkina-Faso (Abonnenc, 1972). Cette répartition est étroitement superposable à celle de la leishmaniose cutanée humaine. Mais si le rôle de vecteur de P. duboscqi dans la transmission de cette affection a été démontré au Sénégal, et si la structure épidémiologique est en partie connue, il est difficile d'extrapoler ces connaissances dans les autres pays.

Les piégeages lumineux, même ceux pratiqués à l'entrée des terriers, n'ont été positifs qu'entre mars et début septembre, ces dates délimitent la période durant laquelle $P$. duboscqi s'éloigne des terriers et transmet la maladie. Nos résultats correspondent à ceux de Dedet et al. (1980) et Dedet \& Desjeux (1982).

La population de la station 1, plus nombreuse, a atteint son maximum dès avril, comme celle de la station 2 . Cette dernière s'est maintenue plus longtemps à un niveau élevé. Cette constatation trouve peut-être sa raison dans les pulvérisations d'insecticides effectuées plus régulièrement dispensées au niveau des vergers et jardins du Monastère.

La courbe d'abondance de l'ensemble des captures présente trois pics bien marqués, qui semblent traduire une succession de trois générations. A la station 2, où l'on rencontre le peuplement le plus dense, ces pics sont nets; les plus importants étant ceux de juillet en 1988 et août 1991. Par contre, à la station 1, le pic maximal d'avril 1988 est précédé d'un premier clocher en février, difficilement explicable, alors que le sommet de juillet n'apparaît pas. Dans la ville même de Dakar, Abonnenc (1972) avait obtenu une courbe de variations présentant un premier clocher en avril et un second en juillet, le troisième n'était pas individualisé.

$P$. duboscqi est connu pour être inféodé aux terriers de rongeurs (Dedet et al., 1980a; Desjeux et al., 1981; Philippe, 1984). Les terriers de rongeurs font figure à la fois de gîtes larvaires et de gites de repos préférentiels pour $P$. duboscqi. En effet, en plus des conditions cli- 
matiques favorables et la présence des rongeurs-hôtes, les déchets de nourriture végétale et les excréments de ces animaux servent de nourriture aux larves.

La présence constante de $P$. duboscqi est notée à l'entrée des terriers, durant toute l'année. La période d'intense activité de $P$. duboscqi débute à partir du mois de décembre avec une augmentation progressive pour aboutir aux plus fortes densités entre mai et août. Cette tendance est probablement liée non seulement à l'augmentation de la température et de l'hygrométrie, mais aussi à l'abondance des rongeurs durant ces mois. D'après Duplantier et Granjon (1992), "la reproduction des rongeurs commence avec les pluies et se poursuit plus ou moins longtemps selon leur intensité et leur répartition ". Dedet et al. (1981) ont mentionné que les femelles de rongeurs sont gestantes en septembre-octobre et les jeunes apparaissent en novembre-décembre. Ainsi, l'abondance de ces animaux augmente le nombre d'appâts disponibles pour $P$. duboscqi, donc le nombre de repas sanguins, puis de pontes. En effet, c'est durant cette période que les femelles gravides ou gorgées sont plus nombreuses. Du fait de la durée du cycle trophogonique et du développement larvaire, on peut supposer que les effectifs de $P$. duboscqi augmenteraient une quarantaine de jours après l'apparition des jeunes rongeurs.

La pluviométrie est impliquée dans la chute brutale observée en juillet-août. Les fortes pluies ont une action directe sur l'abondance de la population de $P$. duboscqi avec la gêne des adultes dans leurs déplacements et une action indirecte avec l'inondation des gîtes larvaires.

Nos études parasitologiques n'ont pas permis de retrouver les résultats des équipes de Dedet et de Desjeux qui ont mis en évidence la présence de Leishmania major dans le tube digestif de six spécimens de P. duboscqi (Dedet et al., 1980a; Desjeux et Waroquy, 1981b). Dedet et al. (1981) ont isolé de trois Mastomys et deux Tatera des souches de Leishmania identiques à celles trouvées chez l'homme et chez $P$. duboscqi.

Le foyer de Keur Moussa est qualifié de sous-type rural zoonotique de leishmaniose cutanée par Desjeux et al. (1982). Il est inféodé aux rongeurs-réservoirs Arvicanthis niloticus, Mastomys erythroleucus et Tatera gambiana. L'hypothèse de fonctionnement formulée est basée sur une variation à long terme liée probablement aux fluctuations des populations de rongeurs. La pullulation de ceux-ci se manifeste par des flambées épidémiques favorisées par l'abondance de la pluviométrie et alternées avec des phases inter-épidémiques durant lesquelles l'endémie se cantonne dans les terriers, le parasite se transmettant par $P$. duboscq $i$ d'un rongeur à un autre. A cela, s'ajoute un rythme saisonnier de la transmission de l'affection à l'homme et l'apparition de cas humains liés à la dynamique des populations de P. duboscqi.

En effet, la première épidémie de 1976-1977 faisait suite à l'énorme pullulation des rongeurs de 1976. Il semble qu'après 1980, le foyer soit entré dans une phase inter-épidémique, aucun cas d'infestation n'ayant été mis en évidence ni chez l'homme, ni chez le vecteur, ni chez les réservoirs (Blanchot et al., 1984). Puis en 1987, une mini-pullulation a été décelée (Duplantier et Granjon, 1992) et une épidémie de leishmaniose l'a aussitôt accompagnée : 79 cas observés entre septembre 1987 et février 1988 par les Sœurs infirmières du dispensaire, sans diagnostic parasitologique.

Depuis cette période, la pluviométrie n'a cessé de décrôtre progressivement $555,5 \mathrm{~mm}$ en 1988, 553 en 1989, 430,4 en 1990 puis 333,5 en 1991; il s'y ajoute une raréfaction des rongeurs. D'autres facteurs s'y sont ajoutés : disparition progressive des Tatera qui vivaient dans les haies périphériques du domaine du Monastère, réduction de la densité des Arvicanthis et Mastomys qui n'ont été rencontrés qu'entre mars et juillet. La population de $P$. duboscqi est ainsi entretenue principalement par l'abondance des Cricetomys, or ceuxci n'ont pas encore été trouvés porteurs de leishmanies et se sont montrés réfractaires aux souches humaines (Larivière, 1966). Les données négatives recueillies dans la recherche d'infestation tendent à montrer que ce foyer se trouve dans une phase interépidémique depuis quelques années. Le parasite, s'il existe encore dans la zone, doit être cantonné dans des îlots de résistance.

Il est nécessaire, pour la surveillance de ce foyer, d'étendre l'étude écologique de la faune phlébotomienne aux habitations du Monastère et au village de Keur Moussa afin de mieux préciser le degré de contact phlébotome-homme. La population de Cricetomys mérite une attention particulière; sa relation avec la population de $P$. duboscqi devant être confirmée par l'analyse des repas sanguins trouvés chez les femelles de ce dernier. Un suivi permanent est à assurer pour ce qui concerne les cas humains après leur confirmation parasitologique.

\section{RÉFÉRENCES}

ABonnenc E. Les Phlébotomes de la région éthiopienne (Diptera, Psychodidae). Mémoires de l'ORSTOM, 1972, 55, 1289.

Blanchot M., Lusina D. \& Beunier E. Surveillance interépidémique d'un foyer de leishmaniose cutanée au Sénégal. Médecine tropicale, 1984, 44, 35-40.

Dedet J.P., Derouin F. \& Cornet M. Infestation spontanée de Phlebotomus duboscqi par des promastigotes de Leishmania au Sénégal. Comptes-Rendus des Séances de l'Académie des Sciences, Paris, série D, 1978, 286, 301-302. 
Dedet J.P., Derouin F. \& Hubert B. Écologie d'un foyer de leishmaniose cutanée dans la région de Thiès (Sénégal, Afrique de l'Ouest). I. Rappel sur la situation de la leishmaniose cutanée au Sénégal et présentation de la zone étudiée. Bulletin de la Société de Pathologie Exotique, $1979 a, 72$ (2), 124-131.

Dedet J.P., Derouin F., Hubert B., Schnur L.F. \& Chance M.L. Isolation of Leishmania major from Mastomys erythroleucus and Tatera gambiana in Senegal (West Africa). Annals of Tropical Medicine and Parasitoloy, 1979b, 72, 333-337.

Dedet J.P., Desjeux P. \& Derouin F. Écologie d'un foyer de leishmaniose cutanée dans la région de Thiès (Sénégal, Afrique de l'Ouest). 4. Infestation spontanée et biologie de Phlebotomus duboscqi Neveu-Lemaire, 1906. Bulletin de la Société de Pathologie Exotique, 1980a, 73, 266-276.

Dedet J.P., Hubert B., Desjeux P. \& Derouin F. Écologie d'un foyer de leishmaniose cutanée dans la région de Thiès (Sénégal, Afrique de l'Ouest). 5. Infestation spontanée et rôle de réservoir de diverses espèces de rongeurs sauvages. Bulletin de la Société de Pathologie Exotique, 1981, 74, 71-77.

Dedet J.P., Lemasson J.M., Martin J., Pradeau F. \& Veys A. La leishmaniose cutanée dans la région du Fleuve (Sénégal, Afrique de l'Ouest). Évaluation du taux d'immunité dans la population humaine. Annales de la Société Belge de Médecine tropicale, 1979c, 59, 21-32.

Dedet J.P., Marchand J.P., Strobel M., Derouin F. \& PraDEAU F. Écologie d'un foyer de leishmaniose cutanée dans la région de Thiès (Sénégal, Afrique de l'Ouest). 2. Particularités épidémiologiques et cliniques de la maladie humaine. Bulletin de la Société de Pathologie Exotique, $1979 d, 72,245-253$.

Dedet J.P., Pradeau F., de Lauture H., Philippe G. \& SanKALE M. Écologie d'un foyer de leishmaniose cutanée dans la région de Thiès (Sénégal, Afrique de l'Ouest). 3. Évaluation de l'endémicité dans la population humaine. Bulletin de la Société de Pathologie Exotique, 1979e, 72, 451461.

Dedet J.P., Saf'Janova V.M., Desjeux P., Emelyanova L.P., Schnur L.F. \& Chance M.L. Écologie d'un foyer de leishmaniose cutanée dans la région de Thiès (Sénégal, Afrique de l'Ouest). 6. Caractérisation et typage des souches de Leishmania isolées. Bulletin de la Société de Pathologie Exotique, 1982, 75, 155-168.

Dedet J.P., Winshall R., Hayes R.D. \& Desjeux P. Les Phlébotomes (Diptera, Psychodidae) de la vallée du fleuve Sénégal. Première mention de Sergentomyia (Parvidens) lesleyae Lewis et Kirck, 1946 en Afrique de l'Ouest. Annales de Parasitologie Humaine et Comparée, 1980b, 55 , 126-133.

Desjeux P. \& Dedet J.P. Écologie d'un foyer de leishmaniose cutanée dans la région de Thiès (Sénégal, Afrique de l'Ouest). 7. Synthèse épidémiologique après cinq années d'observation et hypothèse de fonctionnement. Bulletin de la Société de Pathologie Exotique, 1982, 75, 620-630.

Desjeux P., Derouin F., Waroquy L. \& Dedet J.P. Écologie d'un foyer de leishmaniose cutanée au Sénégal. Structure et hypothèse de fonctionnement. Afrique médicale, 1981, $20,427-432$.
Desjeux P. \& Waroquy L. Étude entomologique de 3000 Phlébotomes (Diptera, Psychodidae) du Sénégal. Infestation spontanée par Trypanosomatidae. Afrique médicale, 1981, 20, 347-352.

Duplantier J. M. \& Granjon L. Les rongeurs du foyer de leishmaniose de Keur Moussa. Dynamique des populations en 1988-1989. Rapport ORSTOM ES/DK, 1992, n 52/92, 23 p.

LARIVIÈre M. Aspects cliniques et épidémiologiques de la leishmaniose cutanée au Sénégal. Bulletin de la Société de Pathologie Exotique, 1966, 59, 83-98.

Larivière M., Basset M., Basset A., Faye I. \& Camain R. Deux nouveaux cas de leishmaniose cutanée au Sénégal. Bulletin de la Société Médicale pour l'Afrique Noire de Langue française, 1964a, 9, 1-3.

Larivière M., Basset A., Pierre J.L., Ranque P., Faye I. \& Camain R. Existence d'un foyer de leishmaniose cutanée dans le cercle de Thiès. Bulletin de la Société Médicale pour l'Afrique Noire de Langue française, 1964b, 9, 285-287.

Larivière M., Camerlynck P., Ranque P. \& Villot M.T. Arvicanthis sp., réservoir de virus naturel possible de Leishmania tropica au Sénégal. Comptes-Rendus des Séances de l'Académie des Sciences Paris, série D, 1965a, 260, 4869-4870.

Larivière M., Camerlynck P., Ranque P. \& Villot M.T., Diagne S. Raybaud N. \& Toury M.F. A la recherche d'un réservoir de virus animal de la leishmaniose cutanée de l'homme au Sénégal : présence de Leishmania chez Arvicanthis sp. Bulletin de la Société Médicale pour l'Afrique Noire de Langue française, 1965 b, 10, 237-238.

MaOuAD M. Aspects cliniques et épidémiologiques de la leishmaniose cutanée au Sénégal (d'après 170 cas observés entre 1964 et 1978 à la consultation clinique dermatologique du CHU de Dakar). Thèse de Doctorat en Médecine, Université de Dakar, 1978, 125 p.

Niang A.A. \& Trouillet J. Phlébotomes du Sénégal. La faune phlébotomienne du campus universitaire de Dakar (Diptera-Psychodidae) Bulletin de la Société Française de Parasitologie, 1993, 11, 151-157.

Philippe G. Le foyer de leishmaniose cutanée de Keur Moussa (étude épidémiologique et clinique). Thèse de Doctorat en Médecine, Université de Dakar, Sénégal, 1978, 89 p.

Ranque P. \& Camerlynk P. Démonstration du pouvoir pathogène pour l'Homme de la souche de Leishmania isolée chez un Arvicanthis niloticus à Piroundary (Sénégal). Inoculation volontaire, auto-observation. Comptes-Rendus des Séances de l'Académie des Sciences, Paris, série D, 1966, 262, 664-665.

Ranque P., Quilici M. \& Camerlynk P. Arvicanthis niloticus (Rongeur, Muridé), Réservoir de virus de base de la leishmaniose au Sénégal. Bulletin de la Société de Pathologie Exotique, 1974, 67, 167-175.

Ranque P., Quilici M. \& Debaene A. Réceptivité d'Arvicanthis niloticus d'élevage vis-à-vis des différentes souches de Leishmania. Médecine Tropicale, 1972, 32, 449-503.

RIOU M. \& ADVIER M. Leishmaniose cutanée contractée au Sénégal. Bulletin de la Société de Pathologie Exotique, 1933, 26, 254-256.

Reçu le 16 avril 1997 Accepté le 13 novembre 1997 


\section{TIRÉS À PART/REPRINTS}

à commander lors du renvoi de l'épreuve/to be ordered when returning the proof

en sus des 50 exemplaires (" Mémoires " et " Notes de recherche ") gracieusement envoyés aux seuls auteurs abonnés.

In addition to the 50 copies of "Original contributions" and "Research notes" sent free of charge to the authors provided they are subscribers

Prix TTC, port compris/Taxes and postage included

À compter du 1er mars 1998/From March 1st, 1998

\begin{tabular}{|c|c|c|c|c|c|c|}
\hline \multirow{2}{*}{ Nombre/Number } & \multicolumn{2}{|c|}{$1-4$ Pages } & \multicolumn{2}{c|}{$5-8$ Pages } & \multicolumn{2}{c|}{ 9-12 Pages } \\
\cline { 2 - 7 } & FF & US\$ & FF & US\$ & FF & US\$ \\
\hline \multirow{2}{*}{50} & 1040 & 179 & 1100 & 230 & 1290 & 272 \\
100 & 1250 & 257 & 1335 & 282 & 1545 & 328 \\
150 & 1505 & 312 & 1605 & 338 & 1860 & 393 \\
200 & 1810 & 375 & 1930 & 443 & 2230 & 468 \\
\hline
\end{tabular}

